\title{
Una revisión sistemática de técnicas de gamification en aplicaciones educativas inteligentes
}

\author{
Humberto Marín Vega ${ }^{1}$, Giner Alor Hernandez ${ }^{1}$, \\ Ramón Zatarain $\mathrm{Cabada}^{2}$, M. Lucía Barrón Estrada ${ }^{2}$ \\ ${ }^{1}$ División de Estudios de Posgrado e Investigación, \\ Instituto Tecnológico de Orizaba, México \\ ${ }^{2}$ División de Estudios de Posgrado e Investigación, \\ Instituto Tecnológico de Culiacán, México \\ humbert_marin@outlook.com, galor@itorizaba.edu.mx \\ \{rzatarain, lbarron\}@itculiacan.edu.mx
}

\begin{abstract}
Resumen. Gamification es el concepto de la aplicación de la mecánica de juego y técnicas de diseño de juegos para involucrar y motivar a las personas a lograr sus metas. El presente trabajo tiene como objetivo hacer una revisión de las técnicas gamification implementadas en el desarrollo de aplicaciones educativas y la implementación o interacción con otras tecnologías como es el caso de Serious games. La importancia de su investigación es debido a que gamification es un término genérico informal para el uso de elementos de juego en sistemas que no son de juego que tienen como objetivo mejorar la experiencia del usuario y la participación de los usuarios. El objetivo de este análisis identificar que atributos de juego y actividades de aprendizaje están presentes en aplicación gamification y juegos serios pero de igual manera están presentes en otras tecnologías como es el caso de ITIL y TEL
\end{abstract}

Palabras clave: Atributos del juego, categorías de juego, gamificación, juegos serios.

\section{A Systematic Review of Gamification Techniques in Intelligent Educational Applications}

\begin{abstract}
Gamification is the concept of applying game mechanics and game design techniques to engage and motivate people to achieve their goals. This paper aims to make a systematic review of gamification techniques implemented in the development of educational applications and the implementation or interaction with other technologies such as serious games. The importance of this research is because gamification is an informal generic term for the use of game elements in systems in order to improve the user experience and user participation. The aim of this analysis is to identify which game attributes and learning activities are present in gamification-based applications, serious games and other technologies such as ITIL and TEL.
\end{abstract}


Keywords: Game attributes, game categories, gamification, serious games.

\section{Introducción}

En la educación el aprendizaje basado en el juego es un factor motivador, los juegos en el aprendizaje suelen ser atractivos debido a sus reglas, el sistema de recompensas y sus entornos [1]. La motivación en el aprendizaje de los estudiantes tiene un impacto significativo en el rendimiento y los logros de los estudiantes con el aprendizaje basado en el juego debido a que son mejores que los de la enseñanza tradicional, de esta forma se comprueba que el aprendizaje basado en el juego alcanza el objetivo de aprender de manera efectiva [2]. De acuerdo con este progreso en el aprendizaje basado en el juego, se admiten varios aspectos del proceso de aprendizaje, entre los más importantes está en primer lugar, que se anima a los alumnos a elegir una solución o tomar una decisión en un momento determinado. En segundo lugar, los alumnos pueden comprobar cómo el resultado del juego puede cambiar en función de sus decisiones y acciones. En tercer lugar, se anima a los alumnos a ponerse en contacto con otros miembros del equipo para discutir y negociar los pasos posteriores mejorando entre otras cosas sus habilidades sociales [3]. Los aspectos centrales importantes de los juegos incluyen: búsqueda de información, selección de información, desarrollo de estrategias, resolución de conflictos, ejercicio de la toma de decisiones y la negociación. De acuerdo con estos aspectos, el objetivo y la culminación de los juegos es llegar a un consenso en la solución del problema [4].

Gamification es el concepto de la aplicación de la mecánica de juego y técnicas de diseño de juegos para involucrar y motivar a las personas a lograr sus metas [5]. A través de Gamification no sólo podemos crear una mentalidad que anima a los estudiantes a probar cosas nuevas, a no tener miedo de fracasar [6], sino que también permite a los estudiantes a participar en experiencias agradables con el propósito del aprendizaje. Una implementación innovadora para el aprendizaje es el uso de juegos serios (Serious Games, SG) comúnmente motivada por la necesidad de educar, formar o informar sobre un tema determinado [7]. La potencia de los juegos serios como una herramienta para el aprendizaje se basa fundamentalmente en la capacidad de equilibrar el entretenimiento, la interactividad y la re-jugabilidad de los juegos típicos con los objetivos de aprendizaje de un objetivo educativo determinado.

Existen diversas revisiones acerca del gamification como Hamari, Koivisto \& Sarsa [8] que revisaron estudios empíricos sobre gamification, para esto crearon un marco para examinar los efectos de su aplicación mediante la elaboración de definiciones y las motivaciones que esta provoca obteniendo resultados positivos de la aplicación de gamification. Por otra parte Seaborna \& Felsb [9] presentaron un estudio sistemático sobre el uso de gamification en exámenes teóricos y trabajos de investigación relacionados con los sistemas interactivos y los participantes. Diseñaron entendimientos teóricos actuales de gamification y comparaciones con los enfoques relacionados, incluyendo diversos tipos de juegos y el diseño gameful. Los resultados obtenidos muestran que un nivel de conceptualización de gamification creciente en investigaciones con participantes empíricos. Otra revisión en la literatura fue hecha por Fui-Hoon Nah et al. [10] sobre gamification en el contexto educativo y del aprendizaje, en la revisión identifican varios elementos de diseño del juego como puntos, 
niveles/etapas y retroalimentación del progreso del juego para identificar resultados obtenidos en el alumno.

El presente trabajo tiene como objetivo hacer una revisión de las técnicas gamification implementadas en el desarrollo de aplicaciones educativas y la implementación o interacción con otras tecnologías como es el caso de Serious games. La importancia de su investigación es debido a que gamification es un término genérico informal para el uso de elementos de juego en sistemas que no son de juego que tienen como objetivo mejorar la experiencia del usuario y la participación de los usuarios [11]. Además, con este trabajo se pretende ofrecer un enfoque innovador para el aprendizaje debido a las nuevas tecnologías y las nuevas aplicaciones que están surgiendo continuamente.

\section{Estado del arte}

En los últimos años, se han propuesto varios estudios con el objetivo de mejorar la educación bajo el uso de técnicas de inteligencia artificial. La mayor parte de éstos se han centrado en el uso de los juegos serios y aplicaciones gamification en una variedad de contextos. Sin embargo, aún no se reporta el uso de aplicaciones gamification en combinación con otras tecnologías en contextos educativos. En esta sección, se presenta un conjunto de trabajos relacionados con el uso de aplicaciones gamification. Estos trabajos se agruparon de acuerdo con el tipo de tecnología desarrollada: 1) Gamification, 2) Juegos Serios, 3) ITIL y 4) TEL.

\subsection{Gamification}

Simões, Díaz-Redondo, \& Fernández-Vilas [12] encontraron características distintivas de los buenos juegos, con el fin de entender lo que tiene sentido aplicar en los procesos de enseñanza, para esto se utilizó una plataforma online llamada schoooools.com, la cual es un ambiente de aprendizaje colaborativo y social. El objetivo fue extraer los mejores elementos del juego para adaptarlos y utilizarlos en el proceso de enseñanza. Por su parte Armastrong et al. [13] definió el concepto de gamification como una forma de aprovechar mecanismos de motivación para aumentar la inversión individual en un sistema, proceso o recurso; es por ello que se aplicaron estos mecanismos para aumentar el compromiso con el catálogo de una editorial, la implementación de gamification satisface los objetivos de negocio y de audiencia. Una forma de aplicarlo es por medio de la motivación extrínseca en donde se participa únicamente a causa de factores externos no relacionados con la naturaleza de la actividad en sí, tales como la oportunidad de ganar un premio o mediante la amenaza de un castigo. Lubin et al. [14] propusó que gamification ofrece una forma didáctica distinta de diseñar una oportunidad para atraer personas y hacer relevante una situación o instrucción a través de la implementación de gamification en entornos de formación educativa. Por su parte Kapp et al. [15] enfatizó la gamification como una forma de ofrecer una mejora del aprendizaje, si gamification se aplica correctamente podría revolucionar el enfoque de una organización a la formación y el desarrollo. Los beneficios podrían incluir más productividad, menos costos de los procedimientos y la satisfacción general con las iniciativas de formación y desarrollo de la institución u 
organización. Robson, Plangger, Kietzmann, MacCarthy \& Pitt [16] discutieron cómo gamification mejora la forma en que las empresas atraen a los clientes y empleados siguiendo un enfoque Business Horizons basado en los principios de gamification para atraer nuevos clientes, propiciar la participación de los empleados en actividades de gamification todo esto con un control de los casos en el cual también se incluyen las experiencias sin éxito.

\subsection{Serious games}

Obikwelu \& Read [17] determinó el grado en que los juegos serios han adoptado el principio pedagógico del constructivismo en el enfoque del aprendizaje. El objetivo del constructivismo en los juegos serios es que los estudiantes sean capaces de completar el aprendizaje de una manera independiente. Pereira et al. [18] estableció una taxonomía para analizar la situación actual (tendencias y deficiencias), las barreras y facilidades para la adopción de juegos serios en el aprendizaje personal, social y ético; la taxonomía es una herramienta fundamental para crear una estructurada instantánea de un estado actualizado, las tendencias y las deficiencias de un campo de interés. La taxonomía tiene dos temas de clasificación de orientación: el área de la actividad y el objetivo del juego. Carrozzinoa, Evangelistaa, Brondia, \& Loren [19] presentaron SONNA, un proyecto de investigación con el objetivo de analizar el impacto de las redes sociales, Web 2.0 y multimedia interactivos como herramientas para el aprendizaje. El proyecto de investigación SONNA propone investigar nuevas metodologías de aprendizaje potenciado por la tecnología. La hipótesis que se presenta es que la experiencia dentro de estos contextos mixtos generaría procesos de aprendizaje diferentes de los producidos en contextos tradicionales. Raybourn et al. [20] presentó un nuevo paradigma para la formación y la educación más eficaz y escalable llamado aprendizaje Transmedia. El aprendizaje Transmedia se define como el sistema escalable de mensajes que representa un relato o una experiencia básica que se desarrolla a partir del uso de múltiples medios de comunicación, este paradigma de aprendizaje ofrece un uso más eficaz de los juegos serios para la formación y la educación. En Barbosa \& Silva [21] se analizó la importancia de Serious Games y se detallan las fases de desarrollo de un juego serio desarrollado para la Web, utilizando la tecnología WebGL. Con esta tecnología, los desarrolladores crean entornos 3D convincentes y juegos de vídeo en 3D que se pueden acceder por casi todas las personas que tiene una conexión a Internet.

\subsection{Technology enhanced learning}

La introducción de Tecnologías para la Educación (TEL) promete una mayor personalización y una mayor productividad, Laurillard et al. [22] examinaron diferentes enfoques para comparar el costo de tiempo de enseñanza de TEL contra los métodos tradicionales, concluyendo que el modelo de costos y beneficios dentro de una institución es la forma más exacta de la comprensión de cómo los profesores utilizan la tecnología para alcanzar el nivel de productividad deseado. Redeker [23] conceptualizó una taxonomía didáctica de objetos de aprendizaje y un enfoque didáctico de metadatos para la facilitación de patrones de navegación de instrucción reutilizables. El objetivo del desarrollo fue implementar estructuras genéricas de navegación basadas en el 
concepto de jerarquía y tipificación didácticas con el fin de admitir la escritura y los procesos de aprendizaje.

\subsection{Information technology infrastructure library}

Chung, Rainer \& Lewis [24] examinaron el impacto de los cuatro componentes de la flexibilidad de la infraestructura de TI (compatibilidad, conectividad, modularidad, y el personal de TI) en la alineación TI-negocio estratégico y el grado en que varias aplicaciones se implementan dentro de una organización. El estudio refuerza la importancia de ITIL para las organizaciones como una fuente de ventaja competitiva sostenible. Vinogradsky [25] enfatizó que ITIL es ampliamente reconocido como un sistema de mejores prácticas para los departamentos de TI y mesas de servicio es por ellos que está siendo adoptado por las organizaciones de todo el mundo, ya que proporciona un marco basado en la práctica que integra y centraliza la gestión de incidencias, gestión de problemas, gestión de archivos de configuración y gestión del cambio dentro de una plataforma de mesa de servicio. Mohamed, Ribière, O'Sullivan \& M. A. Mohamed [26] implementaron ITIL v2.0 en el marco de trabajo de una empresa de gestión de ingeniería a través de principios de gestión de conocimientos incorporados, este marco establece las actividades que son imprescindibles para la mejora de la implementación de ITIL siguiendo con un enfoque de pensamiento para añadir y mantener una ventaja competitiva a través de un enfoque cliente-conciencia y apoyado en el proceso de toma de decisiones a lo largo de la implementación de ITIL, el resultado es un marco de trabajo completo para que las organizaciones alcancen la eficacia y la innovación en toda la implementación.

\section{Análisis}

Actualmente se considera que las aplicaciones tecnológicas que utilizan juegos pueden explorar un objetivo de aprendizaje o formación profesional. [5] Desde la perspectiva de la innovación, conceptos tales como los juegos serios y gamification son los más interesantes y valiosos en este dominio. Desde un punto de vista académico, los juegos serios se estudian en diferentes disciplinas, como la informática, psicología, ciencias de la educación o la comunicación. A pesar del crecimiento en el uso de juegos serios, muchas preguntas de investigación siguen siendo exploradas y la literatura sigue siendo dispares, tales como las relativas a su diseño, selección, adopción, uso y evaluación. La gamification se considera hoy en día como un motor esencial de la innovación en este campo [5].

Lameras et al. [27] propuso una clasificación de juegos serios con base en las características de diseño de aprendizaje y las propiedades del juego. Este estudio proporciona orientación y apoyo a los profesores de instrucción y los diseñadores de juegos para diseñar, planificar y utilizar los juegos serios para un tema o módulo completo, de esta manera propone el diseño del aprendizaje como modalidad fundamental en la arquitectura de diseño de juego; los atributos del aprendizaje establecidos en la propuesta son aprendizaje colaborativo, individual, transmisión de información y discusión y argumentación; el estudio también propone diversos atributos de los juegos los cuales se toman como base para establecer las siguientes 
categorías juegos de reglas, juegos de objetivos y opciones, juegos de tareas y retos, juegos de colaboración y competencia y juegos de regeneración/evaluación.

Las actividades de aprendizaje en juegos tiene el objetivo de obtener resultados de aprendizaje establecidos por el instructor. El resultado de algunas actividades se utiliza como inicio para otras personas como resultado del seguimiento en el juego que puede adaptarse durante la ejecución de la actividad de aprendizaje. Una actividad de aprendizaje basado en el juego a diferencia del contenido del juego es el propósito central para trabajar en el diseño del aprendizaje, el cual que tiene raíces históricas en el campo más amplio de diseño instruccional [28].

Lameras et al. [27] percibe que en el juego las actividades de aprendizaje son una acción situada - que está influenciado por las creencias y valores que poseen los maestros como los diseñadores de juegos en contextos concretos de la práctica, como un proceso iterativo emergente que se produce durante y antes a la orquestación de la actividad de aprendizaje en el juego. Para esto describe una clasificación de las actividades de aprendizaje que se presenta a continuación en la tabla 1:

Tabla 1. Tipos de aprendizaje y sus actividades de aprendizaje.

\begin{tabular}{|c|c|}
\hline Tipos de actividades de aprendizaje & Actividades de aprendizaje \\
\hline $\begin{array}{l}\text { Transmisión de información } \\
\text { Son actividades que permiten reflejar } \\
\text { la información en el aprendizaje del } \\
\text { jugador [29][30]. }\end{array}$ & $\begin{array}{l}\text { - } \text { Conferencias, notas de lecturas, diapositivas. } \\
\text { - } \text { - Esquemorización de conceptos. } \\
\text { - Ejemplos } \\
\text { - Declaraciones incompletas } \\
\text { - Resumen } \\
\text { - Audios }\end{array}$ \\
\hline $\begin{array}{l}\text { Individual } \\
\text { Son actividades para desarrollar en } \\
\text { solitario y adquirir conocimiento [27]. }\end{array}$ & $\begin{array}{l}\text { - Web-quest (Búsqueda y recuperación de } \\
\text { información) } \\
\text { - Resolución de ejercicios } \\
\text { - Experimentación científica } \\
\text { - Reflexiones } \\
\text { - Simulaciones } \\
\text { - Modelo } \\
\text { - Juegos de rol } \\
\text { - Investigación } \\
\text { - Determinación de pruebas } \\
\text { - Análisis de la evidencia } \\
\text { - Formulación de pruebas } \\
\text { - Conectar explicaciones sobre conocimientos }\end{array}$ \\
\hline $\begin{array}{l}\text { Colaborativo } \\
\text { Actividades que permiten adquirir } \\
\text { conocimiento involucrando a varios }\end{array}$ & $\begin{array}{ll}\text { - } & \text { Reuniones } \\
\text { - } & \text { Proyectos en grupo } \\
\text { - } & \text { Web-quest en grupo } \\
\text { - } & \text { Rangos y reportes }\end{array}$ \\
\hline
\end{tabular}




\begin{tabular}{|c|c|}
\hline Tipos de actividades de aprendizaje & Actividades de aprendizaje \\
\hline $\begin{array}{l}\text { personas para obtener un resultado en } \\
\text { común [27]. }\end{array}$ & $\begin{array}{l}\text { - } \text { Grupo de estudiantes que se presentan } \\
\text { - } \text { preguntas entre ellos } \\
\text { - } \text { Resolución de problemas en grupo } \\
\text { - Recopilación de datos en grupo } \\
\text { - } \text { Análisis de datos en grupo } \\
\text { - Reflexiones en grupo }\end{array}$ \\
\hline $\begin{array}{l}\text { Discusión y argumentación } \\
\text { Actividades que permiten la } \\
\text { retroalimentación [27]. }\end{array}$ & $\begin{array}{l}\text { Discusiones guiadas (tema de discusión } \\
\text { proporcionado por el maestro) } \\
\text { - Discusiones abiertas (tema de discusión } \\
\text { proporcionado por los estudiantes) } \\
\text { - Opciones: datos sobre los eventos y varias } \\
\text { opciones para hacer comentarios por parte de } \\
\text { los estudiantes. } \\
\text { - Debates. }\end{array}$ \\
\hline
\end{tabular}

Beetham [29] definió la actividad de aprendizaje como una "interacción específica de estudiantes con otras personas que utilizan herramientas y recursos específicos, orientados a resultados específicos". Es por ello la importancia del diseño de las actividades de aprendizaje en el desarrollo de juegos serios y aplicaciones gamification para cumplir con el objetivo por el cual fue desarrollado el juego.

Otro aspecto importante a considerar en los juegos son sus atributos es por ello que son objeto de estudio de varios trabajos como Lundgren \& Bjork [31] que definieron los atributos del juego como una forma de resumir las reglas del juego, aunque deja en duda si solo abarca las reglas que definen la mecánica o abarca características utilizadas en el diseño [27]. Desde una perspectiva educativa Cook [32] interpretó los atributos de juego dando énfasis a las propiedades de retroalimentación al tiempo que reconoce las relaciones entre las normas y los atributos del jugador. Los atributos del juego deben ser considerados en el diseño del juego para garantizar un equilibrio entre los desafíos y las habilidades necesarias para alcanzar los objetivos.

Con base en los atributos de un juego, Lameras [27] los clasificó en categorías de juegos dependiendo de sus atributos relevantes. Se realizó un intento para asignar categorías de juego generales comprendiendo los atributos en el juego que se utilizan para la creación de instancias de atributos de juego en la práctica educativa; por ejemplo, las reglas se realizan a través de puntuación. El autor hace esta clasificación debido a que no hay una taxonomía que clasifique los atributos del juego en categorías específicas, la clasificación la elaboró con el objetivo de ayudar a los diseñadores de juegos y de instrucción. Las categorías que presenta Lameras [27] se describen la tabla 2 , las cuales se basan de acuerdo a la identificación de los atributos de juegos en cada una de ellas:

Tabla 2. Categorías de juegos y los atributos de cada categoría.

\begin{tabular}{|c|c|}
\hline Categorías de juegos & Atributos de juegos \\
\hline Juegos de reglas & - Niveles de puntuación \\
\hline
\end{tabular}


Humberto Marín Vega, Giner Alor Hernandez, Ramón Zatarain Cabada, M. Lucía Barrón Estrada

\begin{tabular}{|c|c|}
\hline Categorías de juegos & Atributos de juegos \\
\hline & $\begin{array}{ll}\text { - } & \text { Niveles de movimiento } \\
\text { - } & \text { Temporizadores } \\
\text { - } & \text { Barras de progreso } \\
\text { - } & \text { Instrucciones del juego incluyendo las condiciones } \\
& \text { de la victoria }\end{array}$ \\
\hline Juegos de objetivos y opciones & $\begin{array}{l}\text { - } \text { Diario de juego } \\
\text { - } \text { Misiones } \\
\text { - } \text { Tarjetas objetivas } \\
\text { - } \text { Historias } \\
\text { - } \\
\text { - } \text { Rompélogos anidados } \\
\text { Avatares / NPCs }\end{array}$ \\
\hline Juegos de tareas y desafíos & $\begin{array}{l}\text { - } \text { NPC descripción de las tareas basadas } \\
\text { - } \text { Barras de progreso } \\
\text { - } \text { Múltiples opciones para elegir } \\
\text { - Tareas de rama } \\
\text { - Rompecabezas } \\
\text { - Puntos de investigación } \\
\text { - Requisitos } \\
\end{array}$ \\
\hline $\begin{array}{l}\text { Juegos de colaboración y } \\
\text { competencia }\end{array}$ & $\begin{array}{l}\text { - Juego de roles } \\
\text { - Colaboración de la comunidad } \\
\text { - Sentido épico } \\
\text { - } \text { Bonos } \\
\text { - Temporizadores } \\
\text { - } \text { Descubrimiento líder } \\
\text { - Concursos } \\
\text { - Puntuación } \\
\text { - Inventarios } \\
\text { - Tablas }\end{array}$ \\
\hline $\begin{array}{l}\text { Juegos de retroalimentación y } \\
\text { evaluación }\end{array}$ & $\begin{array}{l}\text { - } \text { Consejos de juego } \\
\text { - } \text { Personajes } \\
\text { - } \text { Niveles de juego } \\
\text { - } \text { Obtención y pérdida de vidas } \\
\text { - } \text { Barras de progreso } \\
\text { - } \quad \text { Cuadros de mando }\end{array}$ \\
\hline
\end{tabular}

A continuación se presenta una tabla comparativa de los trabajos analizados en este estudio los cuales pertenecen a diversas tecnologías como es el caso de gamification, juegos serios, TEL e ITIL con el objetivo de encontrar qué tipo de actividades de aprendizaje se encuentra en cada uno y qué atributos de juego fueron encontrados en 
cada trabajo y con base en atributo del juego encontrado a qué clasificación del juego pertenece ese trabajo. Cabe mencionar que debido a que son varias tecnologías analizadas no todos los trabajos cumplen los aspectos del análisis.

Tabla 3. Análisis de identificación de la categoría y atributos del juego de los trabajos seleccionados para este estudio.

\begin{tabular}{|c|c|c|c|}
\hline Artículo & Objetivo & $\begin{array}{c}\text { Tipos de } \\
\text { actividades } \\
\text { de } \\
\text { aprendizaje }\end{array}$ & $\begin{array}{c}\text { Categoría / Atributo } \\
\text { del juego }\end{array}$ \\
\hline $\begin{array}{l}\text { Simões, Díaz- } \\
\text { Redondo, \& } \\
\text { Fernández-Vilas [12] }\end{array}$ & $\begin{array}{l}\text { Implementación de una } \\
\text { plataforma online. }\end{array}$ & $\begin{array}{l}\text { Transmisión } \\
\text { de } \\
\text { información }\end{array}$ & $\begin{array}{l}\text { Objetivos y opciones } \\
\text {-Descripción de la } \\
\text { tarea. } \\
\text {-Descripción del } \\
\text { contenido. } \\
\text {-Repetición del } \\
\text { objetivo. }\end{array}$ \\
\hline Armastrong et al. [13] & $\begin{array}{l}\text { Estudio de la } \\
\text { motivación extrínseca. }\end{array}$ & Individual & $\begin{array}{l}\text { Objetivos y opciones } \\
\text {-Misiones. } \\
\text {-Jornadas de juego. } \\
\text {-Niveles de juego. }\end{array}$ \\
\hline Lubin et al. [14] & $\begin{array}{l}\text { Implementación en } \\
\text { entornos educativos. }\end{array}$ & Colaborativo & $\begin{array}{l}\text { Colaboración y } \\
\text { competencia } \\
\text {-Colaboración de } \\
\text { comunidad. }\end{array}$ \\
\hline Kapp et al [15] & $\begin{array}{l}\text { Implementación en la } \\
\text { formación y desarrollo } \\
\text { de organizaciones. }\end{array}$ & $\begin{array}{l}\text { Transmisión } \\
\text { de } \\
\text { información }\end{array}$ & $\begin{array}{l}\text { Tareas y desafíos } \\
\text {-Descripción de la } \\
\text { tarea. } \\
\text {-Descripción del } \\
\text { contenido. }\end{array}$ \\
\hline $\begin{array}{l}\text { Robson, Plangger, } \\
\text { Kietzmann, MacCarthy } \\
\text { \& Pitt [16] }\end{array}$ & $\begin{array}{l}\text { Implementación para } \\
\text { atraer nuevos clientes y } \\
\text { empleados. }\end{array}$ & Colaborativo & $\begin{array}{l}\text { Colaboración y } \\
\text { competencia } \\
\text {-Colaboración de } \\
\text { comunidad. } \\
\text {-Descubrimiento } \\
\text {-Niveles de juego } \\
\text {-Roles }\end{array}$ \\
\hline Obikwelu \& Read [17] & $\begin{array}{l}\text { Determinación del } \\
\text { grado de adoptado en el } \\
\text { principio del } \\
\text { constructivismo en un } \\
\text { enfoque educativo. }\end{array}$ & Colaborativo & $\begin{array}{l}\text { Colaboración y } \\
\text { competencia } \\
\text {-Colaboración de } \\
\text { comunidad. }\end{array}$ \\
\hline
\end{tabular}


Humberto Marín Vega, Giner Alor Hernandez, Ramón Zatarain Cabada, M. Lucía Barrón Estrada

\begin{tabular}{|c|c|c|c|}
\hline Artículo & Objetivo & $\begin{array}{c}\text { Tipos de } \\
\text { actividades } \\
\text { de } \\
\text { aprendizaje } \\
\end{array}$ & $\begin{array}{c}\text { Categoría / Atributo } \\
\text { del juego }\end{array}$ \\
\hline Pereira et al. [18] & $\begin{array}{l}\text { Desarrollo de una } \\
\text { taxonomía de objetivos } \\
\text { de aprendizaje. }\end{array}$ & $\begin{array}{l}\text { Transmisión } \\
\text { de } \\
\text { información }\end{array}$ & $\begin{array}{l}\text { Tareas y desafíos } \\
\text {-Descripción de la } \\
\text { tarea. }\end{array}$ \\
\hline $\begin{array}{l}\text { Carrozzinoa, } \\
\text { Evangelistaa, Brondia, } \\
\text { \& Loren [19] }\end{array}$ & $\begin{array}{l}\text { Presenta SONNA, } \\
\text { plataforma para } \\
\text { investigar nuevas } \\
\text { métricas de } \\
\text { aprendizaje. }\end{array}$ & Colaborativo & $\begin{array}{l}\text { Colaboración y } \\
\text { competencia } \\
\text {-Colaboración de } \\
\text { comunidad. } \\
\text {-Tablas de } \\
\text { clasificación. }\end{array}$ \\
\hline Raybourn et al. [20] & $\begin{array}{l}\text { Presenta un nuevo } \\
\text { paradigma de } \\
\text { aprendizaje llamado } \\
\text { Transmedia. }\end{array}$ & Colaborativo & $\begin{array}{l}\text { Colaboración y } \\
\text { competencia } \\
\text {-Colaboración de } \\
\text { comunidad. } \\
\text {-Tablas de } \\
\text { clasificación. }\end{array}$ \\
\hline Barbosa \& Silva [21] & $\begin{array}{l}\text { Detalla las fases de } \\
\text { desarrollo de un juego } \\
\text { serio desarrollado para } \\
\text { la web }\end{array}$ & $\begin{array}{l}\text { Transmisión } \\
\text { de } \\
\text { información }\end{array}$ & $\begin{array}{l}\text { Tareas y desafíos } \\
\text { - Descripción de la } \\
\text { tarea. } \\
\text { - Descripción del } \\
\text { objetivo. }\end{array}$ \\
\hline Laurillard et al. [22] & $\begin{array}{l}\text { Modelo orientado a } \\
\text { costos en la enseñanza }\end{array}$ & $\begin{array}{l}\text { Transmisión } \\
\text { de } \\
\text { información }\end{array}$ & $\begin{array}{l}\text { Tareas y desafíos } \\
\text { - Descripción de la } \\
\text { tarea. }\end{array}$ \\
\hline Redeker [23] & $\begin{array}{l}\text { Implementación de } \\
\text { patrones de navegación }\end{array}$ & Individual & $\begin{array}{l}\text { Tareas y desafíos } \\
\text { - Puntos de } \\
\text { investigación. } \\
\text { - Requisitos del } \\
\text { estudio. } \\
\text { - Niveles de juego. }\end{array}$ \\
\hline $\begin{array}{l}\text { Chung, Rainer \& } \\
\text { Lewis [24] }\end{array}$ & $\begin{array}{l}\text { Implementación ITIL } \\
\text { en organizaciones }\end{array}$ & Colaborativo & $\begin{array}{l}\text { Colaboración y } \\
\text { competencia } \\
\text { - Colaboración. } \\
\text { - Clasificación. }\end{array}$ \\
\hline
\end{tabular}

\section{Discusión}

La tabla 3 presenta los trabajos analizados en este estudio sometidos a la clasificación de Lameras [27] tomando en cuenta los tipos de actividades de 
aprendizaje, los atributos de los juegos y la clasificación establecida, los trabajos presentados están elaborados en diversas tecnologías como gamification, juegos serios, Information technology Infrastructure Library y Technology enhanced learning con el objetivo encontrar atributos y tipos de actividades para encontrar un punto de referencia para su posterior integración a gamification.

El objetivo de las actividades de aprendizaje en juegos es obtener un resultado establecido es por ello que se sometió a una evaluación los trabajos previamente seleccionados tomando como base la clasificación de Lameras [27] para identificar dependiendo de las actividades que se desempeñaron en cada uno de ellos; para hacer la clasificación de a que tipo de actividades pertenece cada trabajo y de igual manera analizar si cumplían con el propósito para el que fueron diseñados.

Después de identificar los tipos de actividades de aprendizaje se identificaron los atributos del juego que estaban presentes en cada trabajo, cabe mencionar que aunque no todos los trabajos tienen la aplicación de un juego o alguna técnica relacionada se sometieron al análisis para identificar aquellos factores que estén presentes en su desarrollo para posteriormente como trabajo a futuro integrar las diferentes tecnologías implementadas en aplicaciones gamification con el objetivo de fortalecer su aplicación y abarcar otras tecnologías emergentes.

El resultado de los tipos de actividades de aprendizaje presentes en los trabajos analizados se muestra en la Figura 1.

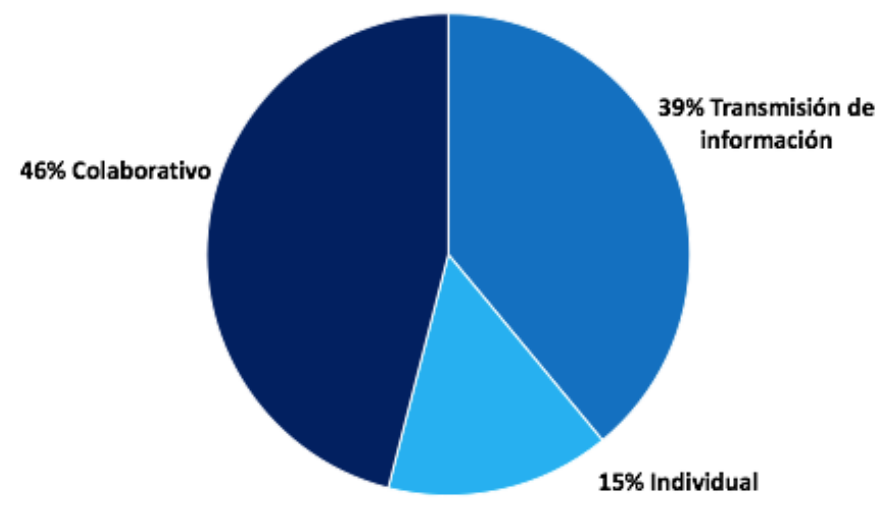

Fig. 1. Tipos de actividades de aprendizaje encontradas en los artículos seleccionados.

El resultado obtenido en trabajos de gamification presentan la transmisión de información, individual y colaborativo dependiendo del contexto de cada trabajo, al igual que los trabajos relacionados con los juegos serios que están presentes estos tipos de actividades de aprendizaje transmisión de información, individual y colaborativo; mientras tanto en las otras tecnologías analizadas como TEL presentó la transmisión de información y actividades del tipo individual e ITIL presentó el tipo de actividad colaborativo. Las aplicaciones gamification y los juegos serios al estar ligados con el uso e implementación de juegos se hizo una identificación de todos los tipos de actividades de aprendizaje ya que pertenecen a la misma área, mientras que TEL e ITIL son tecnologías que aunque no implementan técnicas de juego se identificaron actividades de aprendizaje y atributos del juego para en un futuro por medio de este punto en común se puedan implementar en aplicaciones gamification. 
Los atributos del juego presentes en los trabajos de aplicaciones gamification son la descripción de tareas, descripción de contenido, repetición del objetivo, misiones, jornadas de juego, niveles de juego y la colaboración de comunidad; las aplicaciones gamification emplean técnicas del juego es por ello que se pueden identificar mas de un atributo en cada una de las aplicaciones. En los juegos serios los atributos presentes son la colaboración de la comunidad, tablas de clasificación, descripción de la tarea y descripción del objetivo; los juegos serios son juegos cuyo objetivo esta definido desde su diseño es por ello que la descripción de la tarea y del objetivo están definidas desde su diseño. Por otra parte los trabajos relacionados con TEL presentaron los atributos de juego descripción de la tarea, niveles, requisitos de estudio y puntos de investigación. ITIL presentó la colaboración y clasificación.

Una vez identificados los atributos de los juegos, se realizó una clasificación de acuerdo a los atributos a qué categoria de juego pertenecen los trabajos, estos resultados se observan en la Figura 2.

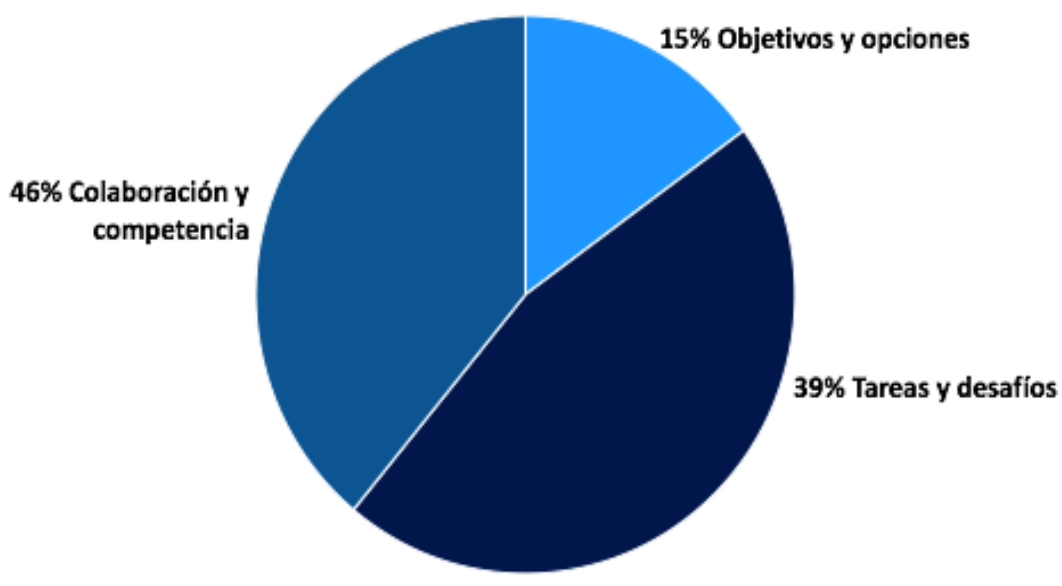

Fig. 2. Categorías de juegos encontradas en los artículos seleccionados.

Como resultado de la revision sistematica se obtuvo que la categoria de juegos que mas se implementa en aplicaciones gamification y juegos serios es la competencia y colaboracion, la cual se implementa en casi la mitad de los trabajos analizados, seguido de la categoria de Tareas y desafios y la categoria de Objetivos y opciones. Cabe destacar que en ninguno de los trabajos se encontraron atributos de juego de la categoria de Discusion y argumentación.

\section{Conclusión y trabajo a futuro}

La implementación de Gamification y juegos serios resulta ser innovadora para el aprendizaje, Gamification es el concepto de la aplicación de la mecánica de juego y técnicas de diseño de juegos para involucrar y motivar a las personas a lograr sus metas proporcionando experiencias agradables con el objetivo del aprendizaje. Mientras que los juegos serios son una herramienta potente para el aprendizaje basados fundamentalmente en la capacidad de equilibrar el entretenimiento, la interactividad y 
la re-jugabilidad con los objetivos de aprendizaje de un objetivo educativo determinado. El presente trabajo presenta una revisión sistemática de trabajos actuales en el área de gamification y juegos serios sometiéndolos a una identificación de los tipos de actividades de aprendizaje presentes en casa trabajo así como que atributos de juego presenta y en base a ellos a que clasificación de juegos pertenece.

Como trabajo a futuro se considera realizar una investigación de más trabajos relacionados con las tecnologías de este estudio con el fin de analizar de manera detallada que otros tipos de actividades de aprendizaje están presentes así como que atributos de juego abarcan. De igual manera se pretende abordar otras clasificaciones de juegos propuestas como la clasificación de Caillois [33], Lavega [34] entre otros. Así como investigar y analizar otros atributos de juego como los propuestos por Caillois [33].

Agradecimientos. Este trabajo es apoyado por el Consejo Nacional de Ciencia y Tecnología (CONACYT), Tecnológico Nacional de México (TecNM) y la Secretaria de Educación Pública (SEP) a través de PRODEP.

\section{Referencias}

1. Prensky, M.: Digital Game-Based Learning. Minnesota: Paragon House Ed edition (2005)

2. Ching-Hsue, C., Chung-Ho, S.: A Game-based learning system for improving student's learning effectiveness in system analysis course. Procedia - Social and Behavioral Sciences Vol. 31, pp. 669-675 (2012)

3. Pivec, M.: Game-based learning in universities and lifelong learning: "UniGame: Social Skills and Knowledge Training" game concept. Special Issue of J.UCS Human Issues in Implementing e-learning technology, Vol. 10, No. 1, pp. 14-26 (2004)

4. Bouras, V., Igglesis, V., Kapoulas, I., Misedakis, O., Dziabenko, A., Koubek. M.: Gamebased learning using web technologies. Journal of Intelligent games and Simulation, Vol. 3, No. 2, pp. 67-84 (2004)

5. Burke, B.: Gartner Redefines Gamification. 4 de Abril de 2014. http://blogs.gartner.com/brian_burke/2014/04/04/gartner-redefines-gamification/ (último acceso: 31 de Marzo de 2016).

6. Chung-Ho, S., Ching-Hsue, C.: A Mobile Game-based Insect Learning System for improving the learning achievements. Procedia - Social and Behavioral Sciences, No. 103 pp. 42-50 (2013)

7. Michael, D., Chen, S.: Serious Games: Games that educate, train and inform. Mason: Cengage Learning PTR (2005)

8. Hamari, J., Koivisto, J., Sarsa H.: Does Gamification Work? - A Literature Review of Empirical Studies on Gamification. Proceeding at 47th Hawaii International Conference on System Science, pp. 3025-3034 (2014)

9. Seaborna, K., Felsb, D.I.: Gamification in theory and action: A survey. International Journal of Human-Computer Studies, Vol. 74, pp. 14-31 (2015)

10. Fui-Hoon Nah, F., Zeng, Q., Rajasekhar-Telaprolu, V., Padmanabhuni-Ayyappa, A., Eschenbrenner, B.: Gamification of Education: A Review of Literature. HCI in Business Vol. 8527, pp. 401-409 (2014)

11. Pereira, P., Duarte, E., Rebelo, F., Noriega, P.: A Review of Gamification for HealthRelated Contexts. Design, User Experience, and Usability, User Experience Design for Diverse Interaction Platforms and Environments, pp. 742-753 (2014) 
12. Simões, J., Díaz-Redondo, R., Fernandez-Villas, A.: A social gamification framework for a K-6 learning platform. Computers in Human Behavior, Vol. 29, pp. 245-253 (2013)

13. Armstrong, D.: The new engagement game: the role of gamification in scholarly publishing. Learned Publishing, Vol. 26, No. 4, pp. 253-256 (2013)

14. Lubin, L.: The Gamification of Learning and Instruction Field Book. New Horizons in Adult Education \& Human Resource Development, Vol. 28, No. 1, pp. 58-60

15. Kapp, K.: Gadgets, games, and gizmos for learning (1st Ed.). San Francisco, CA: John Wiley (2007)

16. Robson, K., Plangger, K., Plangger, J.H., McCarthy, I., Pitt, L.: Game on: Engaging customers and employees through gamification. Business Horizons (Elsevier) Vol. 59, pp. 29-36 (2016)

17. Obikwelu, C., Read, J.: The serious game constructivist Framework for children's learning. Procedia Computer Science, Vol. 15, pp. 32-37 (2012)

18. Pereira, G., Brisson, A., Prada, R., Paiva, A., Bellotti, F., Kravcik, M.: Serious Games for Personal and Social Learning \& Ethics: Status and Trends. Procedia Computer Science, Vol. 15, pp. 53-65 (2012)

19. Carrozzinoa, M., Evangelistaa, C., Brondia, R., Loren, C.: Social Networks and Web-based Serious Games as Novel Educational Tools. Procedia Computer Science, pp. 303-306 (2012)

20. Raybourn, E.M.: A new paradigm for serious games: Transmedia learning for more effective training and education. Journal of Computational Science, pp. 471-481 (2014)

21. Barbosa, A.F.S., Silva, F.G.M.: Serious Games - Design and Development of OxyBlood. Proceedings of the 8th International Conference on Advances in Computer Entertainment Technology, ACE, (2010)

22. Laurillard, D.: Modelling benefits-oriented costs for technology enhanced learning. Higher Education (Springer) Vol. 54, No. 1, pp. 21-39 (2007)

23. Redeker, G.H.J.: An Educational Taxonomy for Learning Objects. Proceedings of the The 3rd IEEE International Conference on Advanced Learning Technologies (ICALT'03), (2003)

24. Chung, S.H., Rainer Jr., R.K., Lewis, B.R.: e Impact of Information Technology Infrastructure Flexibility on Strategic Alignment and Application Implementations. Communications of the Association for Information Systems, Vol. 11, pp. 191-206 (2003)

25. Vinogradsky, V.: The ITIL Framework: And How It Can Improve Your Service Desk. Alloy software, pp. 16-18 (2008)

26. Mohamed, M.S., Ribière, V.R., O'Sullivan, K.J., Mohamed, M.A.: The re-structuring of the information technology infrastructure library (ITIL) implementation using knowledge management framework. VINE Journal of Information and Knowledge Management Systems (VINE), Vol. 38, No. 3, pp. 315-333 (2008)

27. Lameras, P.: Essential Features of Serious Games Design in Higher Education. Society for research into higher education, pp. 3-22 (2015)

28. McLean, P., Scott, B.: Competencies for Learning Design: A Review of the Literature and a Proposed Framework British Journal of Educational Technology, Vol. 42, No. 4, pp. 557$572(2011)$

29. Beetham, H.: Review: Design for learning programme phase 2. . Review of learning design as part of the JISC's Design for Learning programme. Available at: http://www.jisc.ac.uk/whatwedo/programmes/elearningpedagogy/designlearn.a spx. [Acccessed 28 April 2011] (2008)

30. Gunter, G., Kenny, R., Vick, E.: A Case for a Formal Design Paradigm for Serious Games. The Journal of the International Digital Media and Arts Association, Vol. 3, No. 1, pp. 119, doi: citeulike-article-id:9301918 (2006)

31. Lundgren, S., Bjork, S.: Game mechanics: Describing computer-augmented Games in Terms of Interaction. Paper presented at the TIDSE, Available online at: 
Una revisión sistemática de técnicas de gamification en aplicaciones educativas inteligentes

http://www.itu.dk/stud/speciale/worlddomination/files/rikke/rh/speciale/staffa

n_docs/mechanics.pdf [last accessed 23 February 2015] (2003)

32. Cook, D.: What are game mechanics Available at: http://www.lostgarden.com/2006/10/what-are-game-mechanics.html [Last accessed 23 February 2015]: Lostgarden (2006)

33. Caillois, R.: Teoría de los Juegos. $1^{\text {a }}$. Barcelona: Seix Barral (1958)

34. Lavega, P.: La clasificación de los juegos. http://www.praxiologiamotriz.inefc.es/PDF/ clasificacion_juegos_deportes.pdf (2013) 